\title{
INTEGRATION OF DATA RESULTING FROM VARIOUS TYPES OF LUMBAR SPINE EXAMINATIONS
}

Petra Aradi ${ }^{1}$; László Danka ${ }^{2}$; Gabriella Träger ${ }^{3}$; György Lipovszki ${ }^{1}$; Barbara Abonyi ${ }^{4}$

${ }^{1}$ Budapest University of Technology and Economics, Faculty of Mechanical Engineering, Department of

Mechatronics, Optics and Engineering Informatics

${ }^{2}$ Policlinic of Hospitaller Brothers of St. John of God, Department of Orthopedics

${ }^{3}$ Gold Sanitas Kft.

${ }^{4}$ Semmelweis University, Faculty of Physical Education and Sport Sciences, Department of Psychology aradi.petra.bme@gmail.com

\begin{abstract}
Lower back region and lumbar spine in particular can be measured and evaluated with a number of methods and from a number of viewpoints. Measurement method depends essentially on the objective. Tests can be static or dynamic, can target detection of pathological conditions or just evaluate persons with no specific complaints, can be performed once or repeatedly (as follow-up series).

Authors present the development of a complex system that records and stores examination data of various types and formats (image, video, numeric, text, etc.).

Moiré-based virtual imaging method and a mechano-optical handheld scanning device, developed at the Department of Mechatronics, Optics and Engineering Informatics, Budapest University of Technology and Economics (BME MOGI) are used for static examinations. Dynamic tests can be performed with the system (developed also at BME MOGI) that gives 3D information with the help of depth sensors. Conventional examination results, such as static pressure maps obtained with a podoscope, x-ray images, and results of manual measurements (distances, angles, muscle ductility) provide useful information one by one. Measurement protocols help systematic data collection.
\end{abstract}

Integrating measurement results from previously mentioned methods, and utilizing 3D visualization, data storage and processing, increases the amount of information compared to individual pieces of data, and previously unknown connections can be revealed.

The integrated data acquisition and processing system helps organization and systematic processing of data stored in various (file) formats, type (text, image, numerical, etc.) and information content.

Keywords: lumbar spine, lower back, measurement and evaluation, integrated data acquisition, storage and processing

\section{Introduction}

Large parts of the population in so called civilized countries lead an inactive, sedentary lifestyle. This lack of movement, especially that of physical training often results in different illnesses, a number of those involving the musculoskeletal system. Prominent among musculoskeletal ailments is backache, actually it is said to be the second most common type of pain in adults (the most common being headaches). It would also be worthwhile to investigate causes of headaches, 
as some types of them have musculoskeletal origin, like muscle spasms in the upper back and neck. The human back can be divided into three large parts according to the segments of the vertebral column. In this paper authors focus on the bottommost of these parts, the lumbar fragment or lower back.

When any pain, e.g. lower back pain presents, the possible causes have to be explored thoroughly. Tests performed by physicians are complemented by those of physical therapists and other healthcare and sports professionals. There are quite many examination protocols in everyday use, and a large number of supplementary measurement methods are in use as well. Examination or measurement method depends essentially on the objective. Tests can be static or dynamic, can be used to discover suspected pathological conditions, or just evaluate a person who has no specific complaints, and to follow and map results of therapy. One time and repeated tests or measurements are performed, according again to the purpose of the examination.

Evolving methods and measurement devices can very well add information and provide new insights to tests and measurements considered traditional. Authors summarize traditional methods in examining the lower back and present the use of equipment developed in the frame of Gerincőr (SpineGuard) Project at the Department of Mechatronics, Optics and Engineering Informatics. The paper provides insight into the development process of an integrated data storage and processing system for collecting data from tests and measurements that can be related to lower back complaints.

\section{Methods and results}

There are many examinations, tests and measurements involving the evaluation of lumbar spine, and those that aim to map effects of this region on other parts of the body. In our information technology and computer penetrated era, an increasing number of results of such methods are in one of the many digital formats. Traditional methods, such as the use of tape-measures and goniometers also allow some kind of digitalization, the most simple being the use of a computer program like a spreadsheet to manually record data. Various image formats are the result of the use of imaging equipment from medical systems like $\mathrm{x}$-ray, to not yet so widely spread methods (e.g. moiré-based virtual imaging, the use of 3D scanners, depth sensors, just to mention some). Large amounts of numerical data from other measurement devices such as the mechano-optical handheld scanning device is transformed to visual information.

It is a challenge to integrate the ever increasing types and amount of data into a uniform system that provides access to each and every piece of relevant data without bombarding the user with unnecessary information like rows and rows of numbers. When all these data is stored and human-friendly visualization is provided, a further question and task to be solved arises. Would it be possible to explore connections in this diversified multitude of information?

\section{“Conventional" measurements and tests ${ }^{1-3}$}

Each and every measurement method has criteria of reliability, precision and validity, which is extended by the requirement of repeatability, when measurements have to be repeated frequently. 
The lumbar fraction of the spine can be measured in quite a number of ways. To facilitate collection of objective data, such as numbers, images, motion pictures, everything that is relevant in evaluation of lumbar spine and low back status should be given as "objective" data. So, physical examination can give results as distances and angles. Tests can be static, such as the measurement of the length of extremities, length of muscles in different postures, angle of joints. $\mathrm{X}$-rays both in neutral position and in specific postures belong to the group of static tests, too. Ultrasound, CT and MRI give more detailed views and can focus on more aspects, the latter two methods provide additional information with 3D image reconstruction. Foot pressure maps can be recorded either in standing position, or during walking, when the test is dynamic.

The large number of muscles in the pelvic region is responsible not just for the lumbar region, but can cause symptoms in body parts relatively far from this area. Incorrect pelvis position, is not always visible, one has to measure several parameters to confirm it. To explore the effects of incorrect pelvis position, first the direction in which the pelvis left its anatomical place has to be determined. Forward or backward tilt can affect the sagittal curvature of the whole spine, with emphasis on the lumbar section of course, besides it can modify the position of the hip joint. Causes of pelvic tilt have to be examined, too, to determine whether it is caused by pathological issues or stress related posture problems. For example even in healthy people stress can cause unilateral increase of the muscle tone of tensor fasciae latae, thus causing sideward pelvic tilt. Its further consequences are the shortening of quadratus lumborum muscle on the other side as a result of fatigue, with additional muscle reactions that can lead to knee pain as well.

Dynamic tests are used to test movement. Two different examination techniques have to be mentioned, active and passive movement. Range of motion (ROM) compared to other body parts can directly give numerical data, or result can be classified in a manner used in fuzzy rule based systems. Fuzzy membership functions are defined over the possible domain of ROM, and movement can be classified verbally, using qualifiers like normal, less than normal, extremely small, etc. Experts have to define the numerical mapping of these verbal classifiers by setting up a fuzzy rule base system. The presence and intensity of pain can be given by the widely used verbal 0(1)-10 scale, where zero means no pain and 10 is the worst pain the patient has ever experienced, or graphical scales, like the Wong-Baker FACES Pain Rating Scale can be used. Muscles in different spinal ranges can be tested by control exercises, to evaluate their resistance to certain force and their ductility. These tests can be focused on movement, time-span and number of repetitions. Marker based motion capture systems of various physical principles (like infrared imaging) can also be part of the evaluation.

\section{Recently developed equipment}

Measurement devices shown below were developed as part of Gerincőr (SpineGuard) Project as stated in Introduction. One aim is this project is to develop new measurement methods for the human spine, so results can be applied to lower back.

Moiré-imaging is a well-known and quite old optical method. It is used on the human back as a non-invasive method to obtain information on the inner musculoskeletal structure. Modern moiré equipment, such as the one developed at MOGI, use video projectors, digital cameras and a computer integrated into a portable test system. An improvement and derivation from traditional moiré imaging is the use of projected stripes with different colors. This system is 
dubbed as rainbow scanner and after processing images (Figure 1) provides a $2.5 \mathrm{D}$ model of the human back (Figure 2).
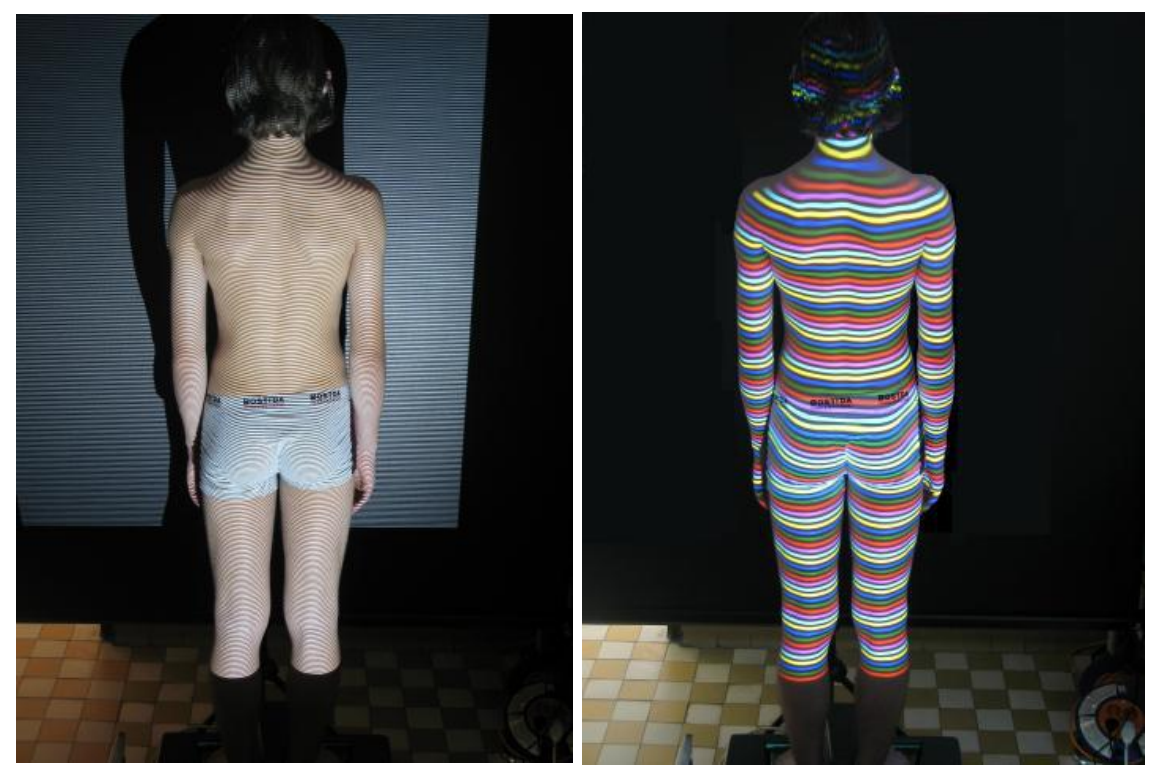

Figure 1. Traditional and rainbow (moiré) scanner setup with stripes projected onto the back

The use of any moiré body scanner requires calibration and standardized measurement conditions, so that results could be compared and evaluated. Methods for scanning and calibration with the rainbow scanner are specified and the processing program was written with these considerations in mind. A large number of school age children were screened for scoliosis at various sites with the rainbow scanner setup, which is equipped with another imaging equipment to simultaneously record foot pressure distribution. Moiré images belong to static measurement, and rely heavily on correct positioning and posture. People using moiré scanners, therefore need to be trained in calibration and examination protocol.

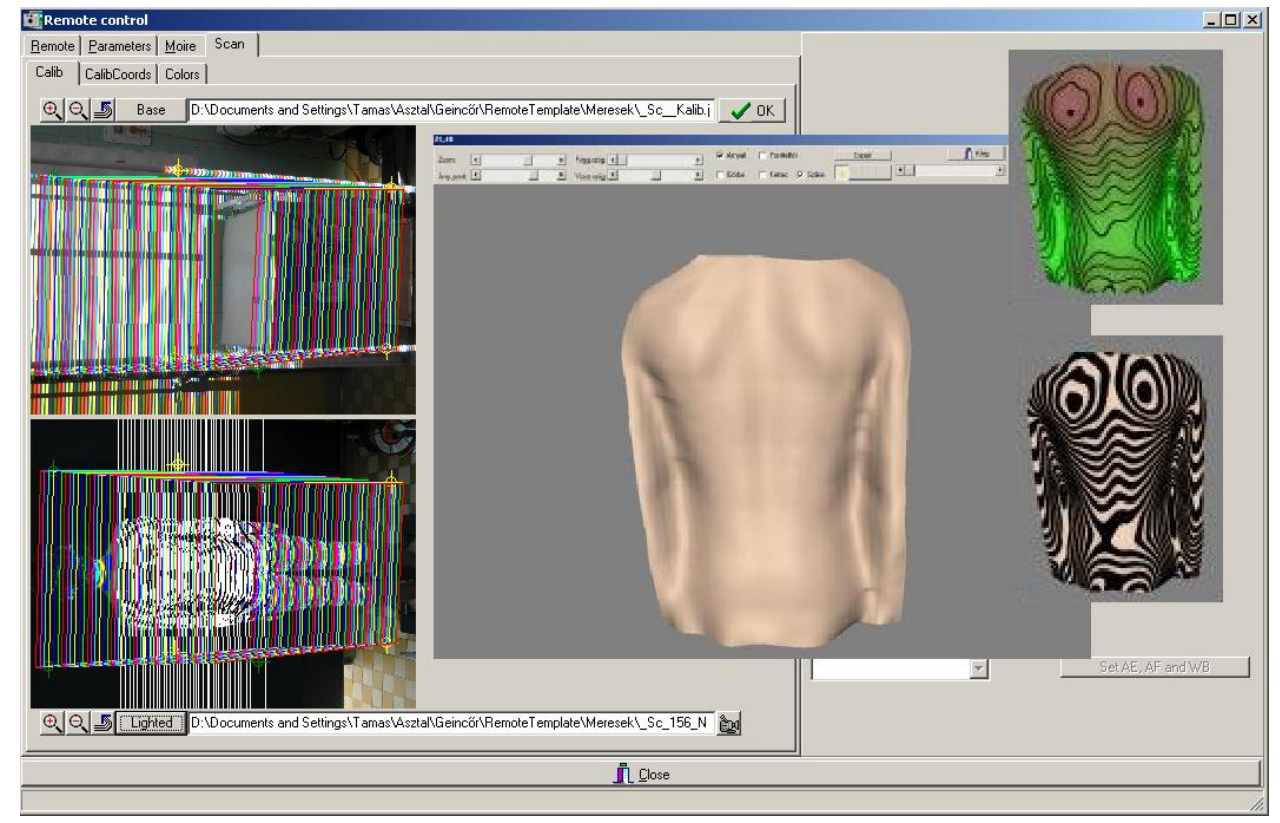

Figure 2. Rainbow (moiré) scanner software with raw image, reconstructed body model and moiré images 
A mechano-optical handheld scanning device, or vertebral scanner for short, has also been developed. The device shows some minimal resemblance to the commercially available SpinalMouse, but has significant differences in measurement principle, measured region and data. After experimenting with various measurement principles, constructions, hardware and software solutions, the device shown in Figure 3 is used now. Data collected along the spine is processed and a 3D geometrical spine model shows the measured position and rotation of each vertebra. The use of this device requires training and the precise following of the measurement protocol, too.
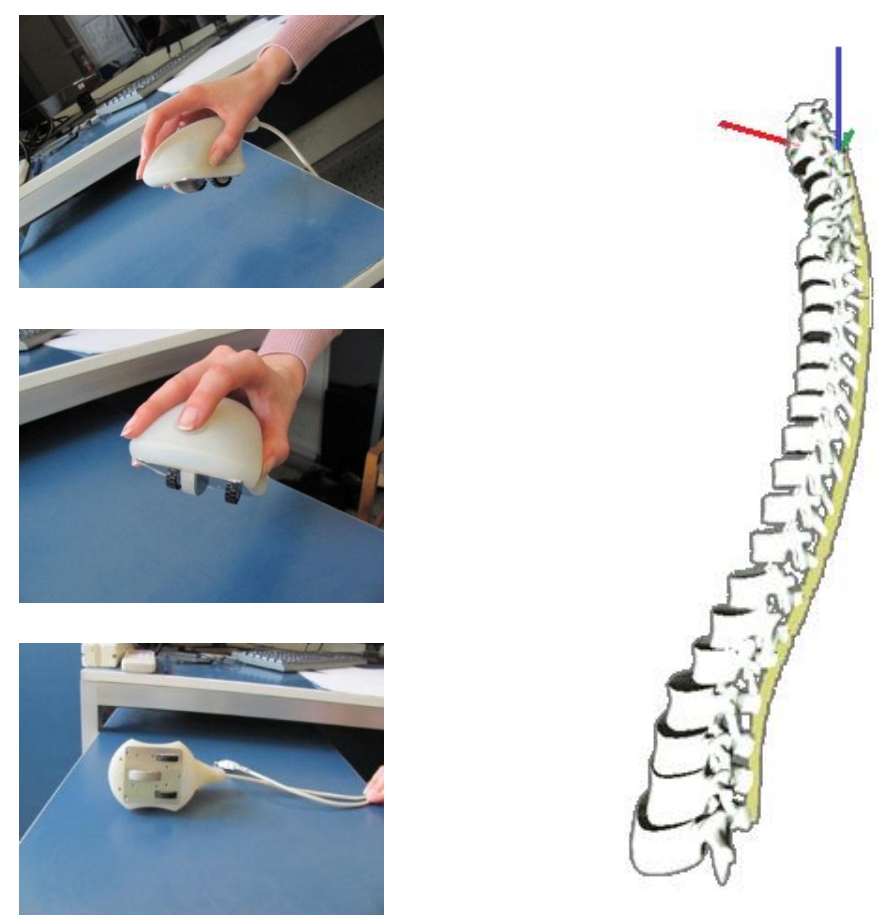

Figure 3. Vertebral scanner, a mechano-optical handheld scanning device and a spine model based on scanned data

The third apparatus uses the depth sensor and cameras built into Microsoft's Kinect. The combination of cameras and depth sensors map 3D objects to $2 \mathrm{D}$ with additional distance information for each pixel. Various graphical representations obtained by the software driving Kinect and processing its data are shown in Figure 4. Depth information also helps create a 3D model of the human body surface.

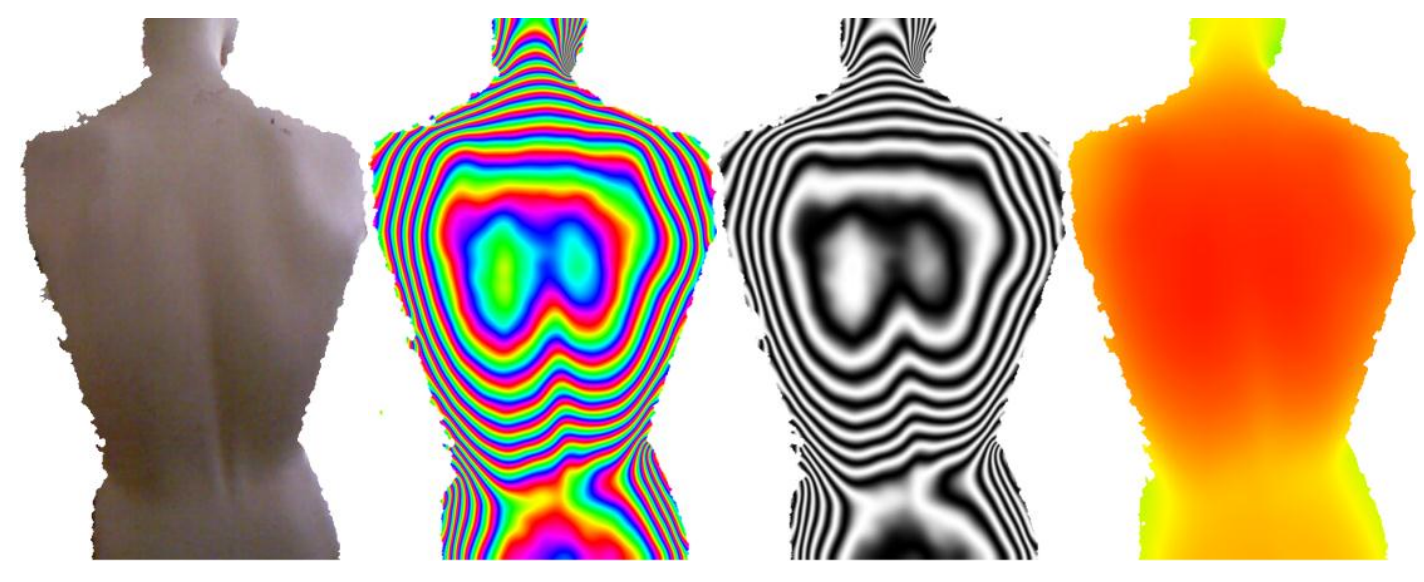

Figure 4. Various representations of the back produced with depth sensors 
The last measurement equipment to be mentioned is the 3D laser body scanner. Person to be scanned has to stand motionless inside a special frame that allows simultaneous movement of laser line sources and taking of multidirectional digital photographs. The associated computer program processes data from these photographs: first it creates the body's point-cloud model, from which it produces a 3D body model with parametric features. Figure 4 shows the pointcloud result of a body scan and two views of the body surface model.
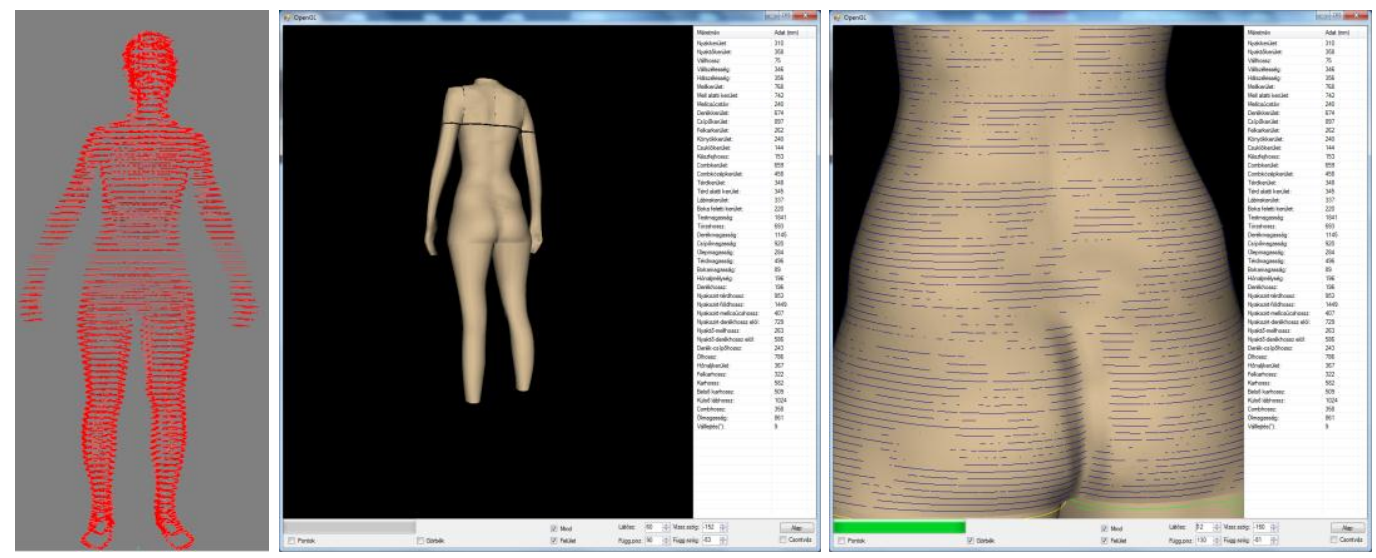

Figure 5. Point-cloud and reconstructed body surface based on data from 3D laser body scanner

\section{Data integration - software structure}

The amount of data is huge in itself. Defining connection among various types of test results require the specification of some (in the magnitude of 100) specific parameters, that represent each data type. These parameters are then used to define connections. Relevant parameters have to be selected by experts, like physicians, physical therapists, etc.

At the time of this writing an XML specification is being created to store relevant data and provide a common connective frame. As various software produce results in different file formats, each program has to be equipped with a data export module. The framework program uses exported XML data from individual software sources and allows users to specify connections. Based on consequences drawn by experts a complex database is under development, enabling access to individual results, and provide follow-up.

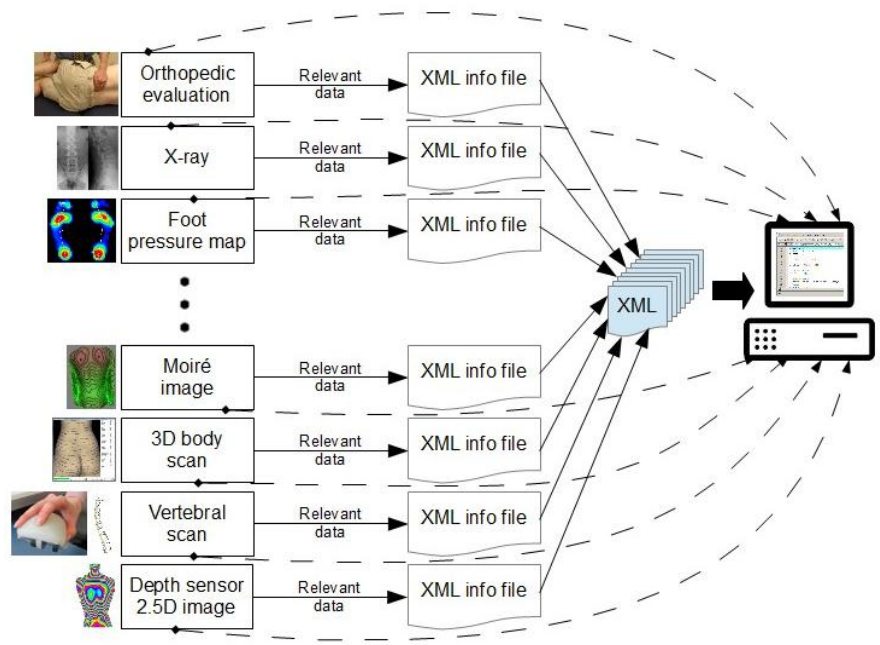

Figure 6. Schematics of collected data, XML info files and program 


\section{Conclusion}

The ever increasing number of tests requires a careful selection; which tests are relevant for the evaluation task in mind. It is also important to check the availability of measurement methods and equipment and their relevance in connecting symptoms, measured data and eventual consequences. When meaningful tests have been chosen, they are repeated, so that progress could be monitored. The amount of gathered data can quite suddenly require adequate storage, which is not the main problem, but it is the platform interconnecting the various results. The developed software aims to provide such a system, focusing to data involving the lower back, at the moment. As more and more data becomes available, the program has to be tuned accordingly; this process is well under way, too.

\section{REFERENCES}

1. Szendrői M (editor). Ortopédia. Budapest: Semmelweis Kiadó, 2009. ISBN: 9789639879492

2. Kocsis L, Kiss RM, Illyés Á. Mozgásszervek biomechanikája. Budapest: Terc Kiadó, 2007. ISBN: 978 9639535640

3. Eston R, Reilly T. Kinanthropometry Laboratory Manual, Anthropometry and Exercise Physiology. Routledge, New York, 2008. ISBN 978-0-415-46671-4

The authors would like to thank National Development Agency (NDA) of Hungarian Government for its support since this study has been cartied out commonly as part of project GERINCO2 TECH_08-A1/2-2008-0121 